\title{
On open mappings, closed mappings, and ordinal invariants
}

\author{
James R. Boone
}

\begin{abstract}
In this brief note new characterizations of open mappings and closed mappings are presented which involve the compact order of the domain. The paper answers some of the questions which resulted from the study of the ordinal monotonicity of pseudoopen mappings and thus constitutes an extension of that work. Another characterization of open mappings is introduced.
\end{abstract}

\section{Preliminaries}

Characterizations of open mappings and closed mappings are presented in terms of the notion of the compact order of a space. This study involves the interaction of ordinal invariants of topological spaces and equations involving the compact closure of the images and preimages of sets. In particular, for a function $f: X \rightarrow Y$ the question of for which ordinals $n$ and $\alpha$ (denoting the $n$th and $\alpha$ th $k$-closures) the equations $(f(A))^{\eta}=f\left(A^{\eta}\right)$ and $f^{-1}\left(B^{\alpha}\right)=\left(f^{-1}(B)\right)^{\alpha}$ are valid is answered in this paper, and provides the characterizations of open mappings and closed mappings.

The following notions were introduced by Arhangel'skiY and Franklin [1]. The k-closure of a set $A \subset X$ is defined as $k \operatorname{cl}(A)=\{p \in X: p \in \operatorname{cl}(A \cap K)$, for some compact $K \subset \ddot{X}\}$.

For each ordinal $\alpha$ the $\alpha$ th $k$-closure is $A^{0}=A$ and $A^{l}=k \operatorname{cl}(A)$, if $\alpha=\beta+1$, then $A^{\alpha}=k \operatorname{cl}\left(A^{\beta}\right)$, and if $\alpha$ is a limit ordinal

Received 4 Septenber 1978. 
$A^{\alpha}=U\left\{A^{\beta}: B<\alpha\right\}$. The compact order of $X$ is

$$
\kappa(X)=\inf \left\{\alpha: A^{\alpha}=\operatorname{cl}(A) \text {, for all } A \subset Z x\right\} \text {. }
$$

For a complete discussion of the properties of compact order see [1], and for a presentation of cardinal invariants see [3]. Recently in [2] the continuous pseudo-open functions were found to be monotonic with respect to the ordinal invariant, compact order. In particular, if $f: X \rightarrow Y$ is a continuous pseudo-open surjection and $X$ is a $k$-space, then $\kappa(X) \geq \kappa(Y)$ [2, Theorem 4.1]. A function $f: X \rightarrow Y$ is said to be $k$-continuous if $f_{K}: K \rightarrow Y$ is continuous for each compact set $K \subset X$. Characterizations of k-continuity which appear in [2, Theorem 3.4 and Corollary 3.5] are as follows.

THEOREM 1.1. Let $f: X \rightarrow Y$. The following are equivalent:

(a) $f$ is k-continuous;

(b) $\left(f^{-1}(A)\right)^{\alpha} \subset f^{-1}\left(A^{\alpha}\right)$, for each ordinal $\alpha$ and each $A \subset Y$;

(c) $f\left(B^{n}\right) \subset(f(B))^{n}$, for each ordinal $n$ and each $B \subset X$.

All statements in this paper are valid for sequential closures, scl, and sequential order, $\sigma$, when the domain is strengthened to a sequential space.

\section{Characterizations}

An open mapping, $f$, is presented in Example 3.1 for which $(f(B))^{\eta} \notin f\left(B^{\eta}\right)$ and $f^{-1}\left(A^{\eta}\right) \notin\left(f^{-1}(A)\right)^{\eta}$, for $\eta<\kappa(X)$. In this example the mapping is not closed and $(f(B))^{\kappa(X)} \ddagger f\left(B^{K(X)}\right)$. Hence pseudo-open mappings do not suffice for the equations to hold, even when the ordinal is $\kappa(X)$. We do have the following:

THEOREM 2.1. Let $f: X+Y$ be a k-continuous pseudo-open surjection, and let $X$ be a k-space. Then $f$ is open if and only if $\left(f^{-1}(A)\right)^{\kappa(X)}=f^{-1}\left(A^{\kappa(X)}\right)$, for each $A \subset Y$.

Proof. $\Leftrightarrow$ Let $p \in f^{-1}\left(A^{\kappa(X)}\right)$. Since $\kappa(X) \geq \kappa(Y)$, $\operatorname{cl}(A)=A^{K(Y)}=A^{K(X)}$. Thus $f(p) \in \operatorname{cl}(A)$. Since $f$ is open, 
$f^{-1}(f(p)) \subset \operatorname{cl}\left(f^{-1}(A)\right)$ or $p \in \operatorname{cl}\left(f^{-1}(A)\right)=\left(f^{-1}(A)\right)^{K(X)}$. Thus $f^{-1}\left(A^{\kappa(X)}\right) \subset\left(f^{-1}(A)\right)^{\kappa(X)}$, and Theorem 1.1 implies

$$
\left(f^{-1}(A)\right)^{\kappa(X)}=f^{-1}\left(A^{K(X)}\right) \text {. }
$$

$\Leftrightarrow$ Let $p \in \operatorname{cl}(A)=A^{\kappa(X)}$. Since $f^{-1}\left(A^{\kappa(X)}\right) \subset\left(f^{-1}(A)\right)^{K(X)}$, $f^{-1}(p) \subset\left(f^{-1}(A)\right)^{\kappa(X)}=\operatorname{cl}\left(f^{-1}(A)\right)$. Thus $f$ is open.

The preceding proof makes use of the following general characterization of open mappings, which was discovered jointly with steve Baber. The surjection $f: X+Y$ is open if and only if for eacin $H \subset Y$ and $p \in Y$ such that $p \in \operatorname{cl}(H), f^{-1}(p) \subset \operatorname{cl}\left(f^{-1}(H)\right)$. This means $f$ is open if and only if $f^{-1}(\operatorname{cl}(A)) \subset \operatorname{cl}\left(f^{-1}(A)\right)$ for each $A \subset y$. This is natural strengthening of the characterization of pseudo-open mappings, in which $p \in \operatorname{cl}(H)$ implies $f^{-1}(p) \cap \operatorname{cl}\left(f^{-1}(H)\right) \neq \varnothing$.

THEOREM 2.2. Let $f: X \rightarrow Y$ be a k-continuous pseudo-open surjection, and let $X$ be a k-space. Then $f$ is closed if and only if $f\left(B^{K(X)}\right)=(f(B))^{\kappa(X)}$ for each $B \subset X$.

Proof. $(\Rightarrow)$ Let $f$ be closed. Then $f\left(B^{K(X)}\right)$ is closed in $Y$ and $f(B) \subset f\left(B^{K(X)}\right)$. Since $\kappa(X) \geq \kappa(Y)$,

$$
(f(B))^{K(X)}=(f(B))^{\kappa(Y)}=\operatorname{cl}(f(B)) \subset f\left(B^{K(X)}\right) .
$$

Thus $(f(B))^{\kappa(X)} \subset f\left(B^{K(X)}\right)$ and Theorem 1.1 implies

$$
f\left(B^{k(X)}\right)=(f(B))^{\kappa(X)} .
$$

$\Leftrightarrow$ Let $B \subset X$. Since $f$ is pseudo-open, $\kappa(X) \geq \kappa(Y)$. Thus

$$
c l(f(B))=(f(B))^{\kappa(Y)}=(f(B))^{K(X)}=f\left(B^{K(X)}\right)=f(\operatorname{cl}(B)) .
$$

Thus $f$ is closed.

\section{Examples}

EXAMPLE 3.1. There is a continuous open mapping $f$ from a sequential space $S_{2}$ onto a convergent sequence $y$ such that $k\left(S_{2}\right)=2$, 
$\kappa(Y)=1,(f(B))^{1} \notin f\left(B^{1}\right), f^{-1}\left(A^{1}\right) \notin\left(f^{-1}(A)\right)^{1}$, and $(f(B))^{2} \pitchfork f\left(B^{2}\right)$.

As in Example 6.2 of [2], let

$$
s_{2}=\{(0,0)\} \cup\left\{\left(\frac{1}{n}, 0\right\}: n \in N\right\} \cup\left\{\left(\frac{1}{n}, \frac{1}{m}\right): n, m \in D\right\}
$$

as in [1] and [3], and let $f$ be the horizontal projection onto $Y=\{(-1,0)\} \cup\left\{\left(-1, \frac{1}{m}\right): m \in N\right\}$ with the usual topology. Let $B=\left\{\left(\frac{1}{n}, \frac{1}{n}\right]: n \in N\right\} \subset S_{2}$. Then $B$ is closed and

$$
f(B)=f\left(B^{1}\right)=f\left(B^{2}\right)=\left\{\left(-1, \frac{1}{n}\right\}: n \in N\right\} \text {. }
$$

Since $(f(B))^{I}=(f(B))^{2}=Y,(f(B))^{I} \notin f\left(B^{I}\right)$ and $(f(B))^{2} \notin f\left(B^{2}\right)$. Let $A=\left\{\left(-1, \frac{1}{n}\right): n \in i v\right\} \subset Y$. Then

$$
f^{-1}\left(A^{I}\right)=f^{-1}(Y)=S_{2} \notin S_{2}-\{(0,0)\}=\left(f^{-1}(A)\right\}^{1} \text {. }
$$

EXAMPLE 3.2. There is a continuous closed mapping $f$ defined on the reals with the usual topology such that $f^{-1}\left(A^{K(R)}\right) \neq\left(f^{-1}(A)\right)^{K(R)}$.

Consider the identification mapping $f: R \rightarrow R / N$. Then

$$
\begin{aligned}
\kappa(R)= & \kappa(R / N)=1 . \text { If } A=(2,3), A^{K(R)}=[1] \cup(2,3) \text {. Thus } \\
& f^{-1}\left(A^{K(R)}\right)=N \cup(2,3) \notin[2,3]=(2,3)^{K(R)}=\left(f^{-1}(A)\right)^{K(R)} .
\end{aligned}
$$

\section{References}

[1] A.V. Arhangel'skiY and S.P. Franklin, "Ordinal invariants for topological spaces", Hichigan Math. J. 15 (1968), 313-320.

[2] James R. Boone, "Pseudo-open functions are monotonic decreasing for ordinal and cardinal invariants", General Topology and Appl. 9 (1978), 243-251.

[3] James R. Boone, Sheldon W. Davis and Gary Gruenhage, "Cardinal functions for k-spaces", Proc. Amer. Math. Soc. 68 (1978), 355-358.

Department of Mathematics, Texas A\&M University, College Station, Texas, USA. 